\title{
Nanofiller Reinforced Polyolefin Elastomer: Effect on Morphology and Mechanical Properties of Composites
}

\author{
Sharmila Pradhan ${ }^{1,2,3}$, Ralf Lach ${ }^{4,5}$, Wolfgang Grellmann ${ }^{4,5}$ amd Rameshwar Adhikari ${ }^{1,2}$ \\ ${ }^{1}$ Central Department of Chemistry \\ Tribhuvan University,Kirtipur, Kathmandu \\ ${ }^{2}$ Nepal Polymer Institute (NPI) \\ Kathmandu \\ ${ }^{3}$ Department of Chemistry, Amrit Science College \\ Lainchour, Kathmandu \\ ${ }^{4}$ Martin-Luther University Halle-Wittenberg, Centre of Engineering \\ Halle/Saale, Germany \\ ${ }^{5}$ Institut für Polymerwerkstoffe e.V., Institute associated with the University of Applied Science \\ Merseburg \\ Merseburg, Germany \\ e-mail: nepalpolymer@yahoo.com
}

\begin{abstract}
The effect of different types of fillers on morphology and mechanical properties of polymer nanocomposites has been investigated using ethylene-1-octene copolymer (EOC), a polyolefin based elastomer, as matrix and various nanofillers \{such as multi-walled carbon nanotubes (MWCNT), layered silicate (LS) and boehmite (OS2)\}. The morphological structures were studied by scanning electron microscopy (SEM) while the mechanical properties were characterized by tensile testing and microindentation hardness measurements. It has been shown that the nature of the nanofiller may have significant influence on the mechanical properties of the samples. Among the nanocomposites studied so far, the MWCNT filled samples showed the highest reinforcing effect followed by layered silicate. The least reinforcing effect was obtained for the samples filled with boehmite nanoparticles.
\end{abstract}

Key words: polyolefin elastomer, nanocomposite, microhardness, mechanical properties

\section{Introduction}

In recent years, many materials scientists are involved in generating multifunctional engineering materials possessing novel properties with the addition of nanosized filler to overcome the disadvantages of traditional composites. The novel polymeric materials incorporated with fillers having ultrafine phase dimensions typically of less than $100 \mathrm{~nm}$, which are especially termed as polymer nanocomposites (PNCs), have attracted special research interest (Alexandre et al. 2000, Biswas et al. 2009). In such materials, through the variation in particulate dimension from micrometer to nanometer scale, the surface area to volume ratio has been found to alter by three orders of magnitude leading to the drastic changes in morphological features as well as in their properties (Powell et al. 2006).
Generally, the new PNCs have been synthesized with three categories of reinforcing materials such as particles (e.g., silica, metal and other organic and inorganic substances), layered materials (e.g., graphite, layered silicate, etc) and fibrous materials (e.g., nanofibres and nanotubes) (Ray \& Okamoto et al. 2003, Tsai \& Sun et al. 2004). The material properties are found distinctly dependent upon the size of the reinforcement particles as well as in the properties of the interphase. Compared to conventional counterparts, these nanocomposites have been found to possess promising mechanical properties such as hardness, tensile modulus, strength and toughness at both low and high temperatures. In addition, the PNCs are found to have significantly improved barrier properties, thermal stability and extinguishing characteristics with advantage of light weight of the 
common polymers (Alexandre et al. 2000). Thus, these are used as excellent prospective materials for food packaging, membranes, adhesives, automotive parts, textiles etc. (Thostension et al. 2005).

The literature review reveals the role of intercalated and interphase volume on the physical properties of the PNCs (Ash et al. 2004, Moczo et al. 2008). In case of the presence of weak particle/matrix interface, the mode of plastic deformation in glassy polymers changes from cavitation to shear yielding leading to a transition from brittle to ductile behavior (Kim 2007). The change has been attributed to the increased polymer chain mobility, presence of smaller particles, and also the capability to relieve triaxial stress because of poorly bonded larger particles (Powell \& Beall 2006). The large surface area of the nanofillers results in large volume fraction of interfacial matrix material with properties entirely different from the bulk polymer. The interfacial area creates significant volume fraction of interfacial polymer even at low loadings ( $<5$ vol.$\%)$. The thermal, mechanical and electrical properties of the composites have also been affected directly with interfacial polymer (Schadler et al. 2007). It has been pointed out that the structure and properties of interfacial polymer is the controlling factor for changes in crystallinity, mobility, chain conformation, chain entanglement, density and charge distribution of thermoplastic polymer based PNCs (Ward \& Sweeney 2004).

Mechanical properties are interdependent upon molecular structure, morphology and processing methods (Giannellis 1996).

The aim of this work is to study the morphology and mechanical properties of the composites based on ethylene-1-octene copolymer elastomer and different kinds of nanofillers.

\section{Methodology}

\section{Materials and sample preparation}

Ethylene-1-octene copolymer (EOC), a commercial product of Dow Chemical Company (trade name: Affinity EG8150) having molecular weight of $161,400 \mathrm{~g} / \mathrm{mol}$, and melt flow index (MFI) of $0.5 \mathrm{~g} /$ min was used as the basic polymer. The degree of crystallinity of the EOC is $16 \%$ and comonomer content is $39 \%$.
Various types of nanofillers such as multiwalled carbon nanotubes (named as MWCNT in this work) manufactured by Bayer Materials (commercial name: Baytubes), organically modified layered silicate (named as LS in this work) developed by Südchemie (commercial name: Nanofil 5) and boehmite nanoparticles manufactured by Sasol Chemicals (named as OS2 in this work), respectively were used.

EOC nanocomposites containing 2 and 5 wt.-\% of various nanofillers were prepared by melt mixing followed by compression molding. Melt mixing was carried out in an internal mixture maintained at $90^{\circ} \mathrm{C}$ and torque of $50 \mathrm{rpm}$ for 10 minutes. Then the mixture was compressed to the sheets of $1 \mathrm{~mm}$ thickness at a temperature of $120{ }^{\circ} \mathrm{C}$ and pressure of 110 bar. Tensile specimens (type 5A according to ISO 527) with length of $75 \mathrm{~mm}$ were punched out of the sheets.

\section{Characterization of nanocomposites}

Scanning Electron Microscopy (SEM): The morphology of the nanocomposites was characterized by scanning electron microscope (SEM) JSM 6300 (JOEL) by using back scattered electron (BSE) imaging modes. Each specimen was cryofractured and sputter-coated with approximately $10 \mathrm{~nm}$ thick layer of carbon film prior to SEM imaging. The contrast in the BSE images directly correlates with the difference in the atomic mass of the elements present and hence represents the materials contrast (Michler 2008).

Tensile Testing: The tensile stress-strain curves of each nanocomposite of EOC was recorded using dog bone shaped specimen by Zwick Z020 universal tensile tester (Zwick/Roell Co., Germany). The measurement was carried out at $23^{\circ} \mathrm{C}$, at the crosshead speed of $50 \mathrm{~mm} / \mathrm{min}$.

Microindentation Test: The microindentation measurements were performed on a Fischerscope H100C recording microhardness tester equipped with a pyramidal Vickers diamond indenter (Helmut Fischer Co., Germany), which was penetrated into the sample with the application of force up to $300 \mathrm{mN}$ at room temperature $\left(23^{\circ} \mathrm{C}\right)$. The loading rate of both loading and unloading was constant to be $15 \mathrm{mN} / \mathrm{s}$. The evaluation of load $(P)$ versus indentation depth $(h)$ curves permitted the determination of different 
hardness parameters such as Martens hardness and indentation modulus (Lach et al. 2010, Bhandari et al. 2012).

\section{Results and Discussion}

\section{Microscopic characterization of PNCs}

Scanning electron microscopy (SEM) was used to inspect the dispersion of the nanoparticles in the polymer matrix. The scanning electron micrographs of the PNCs containing 5 wt.-\% of three different kinds of fillers are shown in Fig. 1.

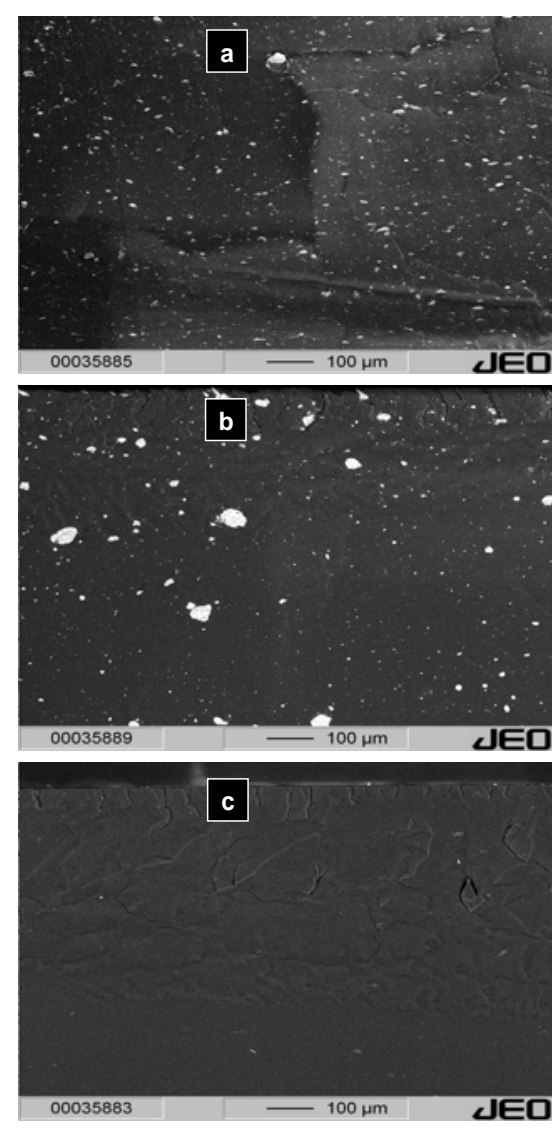

Fig. 1. SEM micrographs of the nanocomposites comprising 5 wt.-\% of different nanofiller: a) boehmite (OS2), b) layered silicate (LS) and c) multiwalled carbon nanotube (MWCNT)

The Fig. 1a shows the back scattered electron imaging of the nanocomposites comprising 5 wt.-\% of OS2. The SEM image of the sample shows clear dispersion of OS2 nanoparticles in the form of white dots. The boehmite particles of several micrometers in diameter are distinctly visible. The SEM images presented in Fig. 1 show the uniform distribution of the fillers with wide variation of particle diameter. The particles with nanometer dimension can be, however, not visible due to limited resolution of the SEM.

The Fig. 1b shows the fracture surface morphology of the PNC containing 5 wt.-\% LS. The SEM imaging shows well dispersed LS particles in the form of fine white dots. Here, the particle diameter is narrower than the PNC with the boehmite nanoparticles indicating better compatibility of LS with the polymer. Figs. 1a and $1 \mathrm{~b}$ show clearly the agglomeration of the nanoparticles albeit the uniform distribution of the aggregates.

The SEM micrograph of EOC consisting of 5 wt.-\% MWCNT depicted in Fig. 1c shows the dark surface of the polymer, in which the nanoparticles could not be observed indicating that MWCNT and polymer has no mass contrast between the inorganic filler and carbon present in the polymer. Nevertheless no agglomerates are visible implying the good dispersion of the nanofiller.

\section{Tensile properties of PNCs}

Fig. 2a compares the stress-strain curves of nanocomposites having different weight fraction of boehmite with pristine EOC. The curves of all the samples in this case are almost identical indicating that the boehmite could not reinforce the tensile property though it is well dispersed in the matrix (see Fig. 1a). It seems that the boehmite could not make the chemical linkages with EOC and/or exhibits only little physical interplay with the macromolecules. The stress-strain curves of virgin polymer and EOC filled with different weight fraction of layered silicate are presented in Fig. 2b. The curves presented are not much different, however, tensile strength is found to be increased slightly with the filler content. Thus, the reinforcing effect of layered filler is found to be higher than that of spherical shaped filler.

The tensile curves of composites containing different weight fractions of MWCNT (see Fig. 2c) shows the decreasing trend of strain at break with filler content but the decrease is not significant. However, it is evident from Fig. 2c that the stress at every strain is found to be increased with the amount of particles in 
the nanocomposites implying. Thus, the MWCNT, cylindrical shaped nanofiller is found to possess most effective reinforcing property in the nanocomposites.
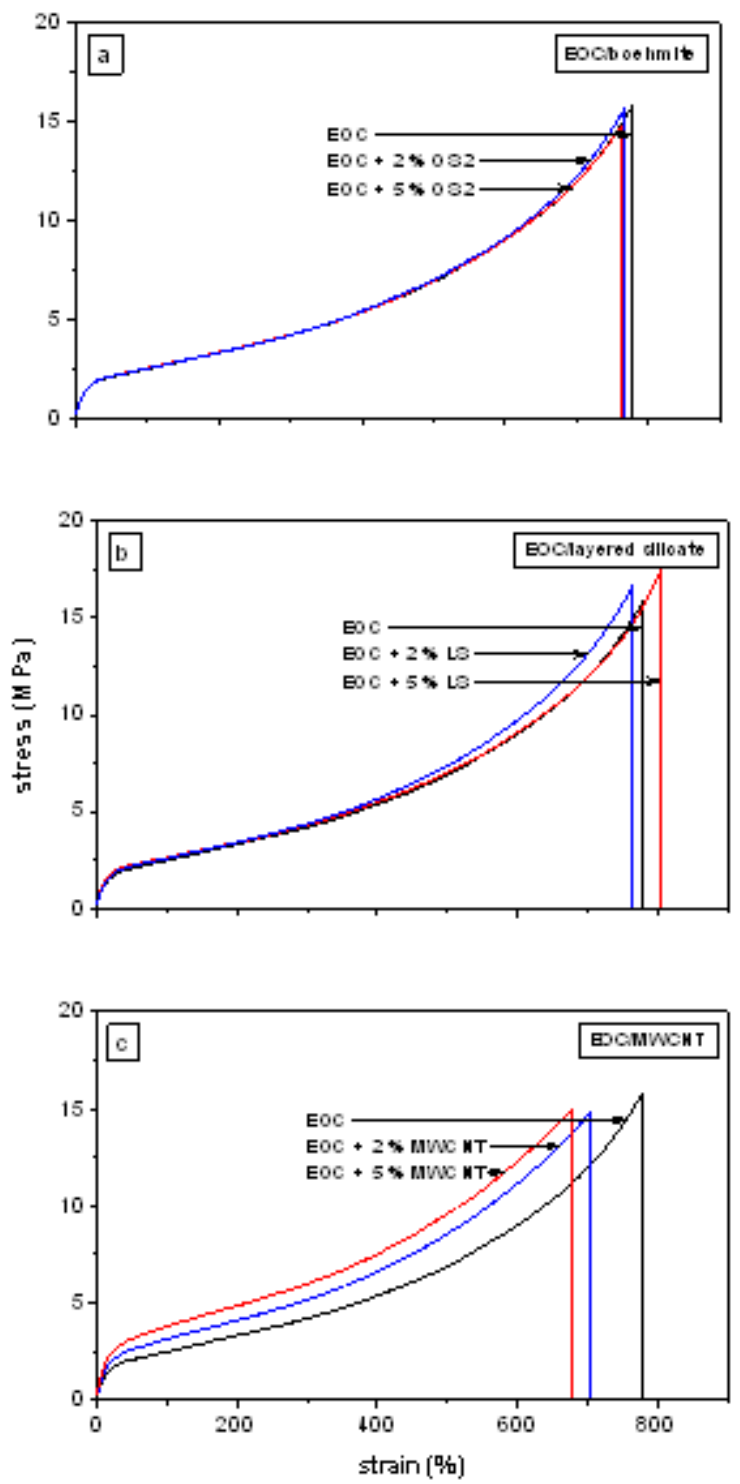

Fig. 2. Stress-strain curves of PNCs consisting of varied weight fraction of different fillers: a) PNCs comprising OS2, b) PNCs with LS and c) PNCs with MWCNT

\section{Microhardness measurement of PNCs}

The microhardness and the elastic properties were determined by recording microindentation measurements (Lach et al. 2010).
The averaged load $(P)$ versus corrected indentation depth $(h)$ diagrams of pure EOC and EOC/OS2 nanocomposites comprising different weight fractions of boehmite is presented in Fig. 3a which shows the identical curve patterns. However, the initial slope of the unloading curves of EOC/OS2 composites having varied weight fractions of OS2 appears lesser than that of virgin EOC curve. Hence, the addition of boehmite results in negative impact in the microindentation hardness of the polymer.
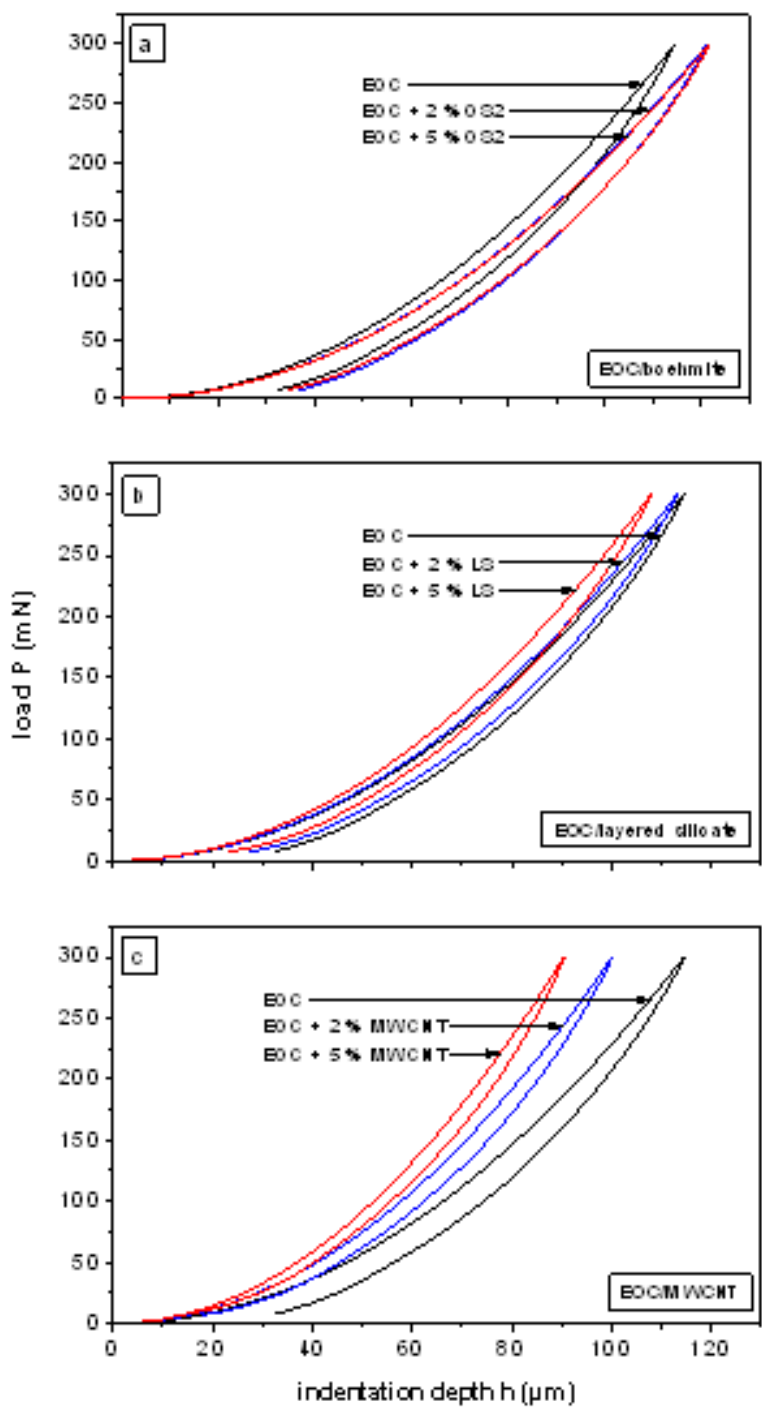

Fig. 3. $P-h$ diagrams of nanocomposites as a function of nanofiller weight fraction: a) EOC/OS2, b) EOC/LS and c) EOC/MWCNT 
The $P-h$ curves of the EOC/layered silicate nanocomposites are presented in Fig. 3b. The experiments were carried out under identical conditions as in the sample presented in Fig. 3a. A closer view of plots of pure EOC and EOC modified with varied amount of LS reveals slight increment in the microhardness behavior of LS filled EOC samples.

In the similar manner, Fig. $3 c$ represents mean $P-h$ curves of pristine EOC and EOC/MWCNT nanocomposites comprising multiwalled carbon nanotubes (MWCNT) of different weight fractions. Fig. 3c clearly shows that the initial slope of the EOC/ MWCNT comprising 5 wt.- $\%$ nanocomposites is higher than that of EOC/MWCNT containing 2 wt.-\% and pure EOC sample. Hence, the addition of varied fractions of MWCNT reinforces the matrix polymer (i.e., EOC) significantly.

The nanocomposites of the polyolefin based ethylene1-octene copolymer (EOC) and different kinds of nanofillers were successfully fabricated. Subsequently, the morphology and mechanical properties of the composites were characterized. The results can be summarized as follows:

1) Each filler type was dispersed in the polymer matrix quite homogeneously. The composites were not exclusively nanocomposites as several micronsized particles were also present. The best compatibility between matrix and filler with respect to the filler size reduction and dispersion was observed in the composites with the LS which may be attributed to the presence of organic modifier intercalating into the layer galleries.

2) The results from tensile testing and microindentation hardness measurements demonstrate the following reinforcing ability of the fillers: MWCNT > layered silicate (LS) > boehmite. The higher reinforcing effects of MWCNT and LS may be attributed to their inherent high aspect ratio.

\section{Acknowledgements}

S. P. acknowledges the German Research Foundation (DFG) for supporting her research stay at MartinLuther University Halle-Wittenberg, Germany. R. A. thanks the Alexander von Humboldt (AvH) Foundation for funding his short term stay at Martin-Luther University Halle-Wittenberg, Germany. Nepal Academy of Science and Technology (NAST) is thanked for providing the $\mathrm{Ph}$. D. research grant for S. P.

\section{References}

Alexandre, M.P. and P. Dubois. 2000. Polymer-layered silicate nanocomposites, preparation, properties and uses of a new class of materials. Material Science and Engineering 28:1-63.

Ash, B. J., R.W. Siegel and L.S. Schadler. 2004. Mechanical behavior of alumina poly(methyl methacrylate) nanocomposites. Macromolecules 37:1358-69.

Bhandari, N. L., R. Lach, W. Grellmann and R. Adhikari. 2012. Depth-dependent indentation microhardness studies of different polymer nanocomposites. Macromolecular Symposia 315:44-51.

Biswas, A., A. Bandyopadhyay, N.K. Singh and A.K. Bhowmick. 2009. Ionomeric modification of metallocenebased polyolefinic elastomers with varied pendant chain length and its influence on physico-mechanical properties. Journal of Material Science 44:3125-3134.

Gianellis, E. P. 1996. Polymer layered silicate nanocomposites. Advanced Materials 8:29-35.

Kim, G.-M. 2007. Reinforcing Mechanisms on the Macro , Micro and Nanoscale in Heterogeneous Polymer Materials. Paperflieger, Clausthal-Zellerfeld, Germany (in German).

Lach, R., G.H. Michler and W. Grellmann. 2010. Microstructure and indentation behaviour of polyhedral oligomeric silsesquioxanes-modified thermoplastic polyurethane nanocomposites. Macromolecular Materials and Engineering 295:484-491.

Michler, G.H. 2008. Electron Microscopy of Polymers. Springer Verlag, Heidelberg, Germany.

Moczo, J. and B. Pukanszky. 2008. Polymer micro and nanocomposites: Structure, interactions, properties. Industrial and Engineering Chemistry 14:535- 563.

Powell, C.E. and G.W. Beall. 2006. Physical properties of polymer/clay nanocomposites. Current Opinion in Solid State and Materials Science 10:73-80.

Ray, S.S. and M. Okamoto. 2003. Polymer/layered silicate nanocomposites; a review from preparation to processing. Progress in Polymer Science 28:1539-641.

Schadler, L.S., L.C. Brinson and W.G. Sawyer. 2007. Polymer nanocomposites: A small part of the story. JOM March 50-58.

Thostension, E.T., L. Chunyu and T.W. Chou. 2005. Nanocomposites in context. Composites Science and Technology 65:491-516.

Tsai, J. and C. T. Sun. 2004. Effect of platelet dispersion on the load transfer efficiency in nanoclay composites. Composite Materials 38:567-79.

Ward, I.M. and J. Sweeney. 2004. An Introduction to the Mechanical Properties of Solid Polymers. John Wiley, Chichester, UK. 
Nepal Journal of Science and Technology Vol. 13, No. 2 (2012) 103-108 